\title{
Fluxon bunching in supercurrent-coupled Josephson junctions
}

\author{
Grønbech-Jensen, Niels; Lomdahl, Peter S.; Samuelsen, Mogens Rugholm
}

Published in:

Physical Review B

Link to article, DOI:

10.1103/PhysRevB.48.6353

Publication date:

1993

Document Version

Publisher's PDF, also known as Version of record

Link back to DTU Orbit

Citation (APA):

Grønbech-Jensen, N., Lomdahl, P. S., \& Samuelsen, M. R. (1993). Fluxon bunching in supercurrent-coupled Josephson junctions. Physical Review B, 48(9), 6353-6359. https://doi.org/10.1103/PhysRevB.48.6353

\section{General rights}

Copyright and moral rights for the publications made accessible in the public portal are retained by the authors and/or other copyright owners and it is a condition of accessing publications that users recognise and abide by the legal requirements associated with these rights.

- Users may download and print one copy of any publication from the public portal for the purpose of private study or research.

- You may not further distribute the material or use it for any profit-making activity or commercial gain

- You may freely distribute the URL identifying the publication in the public portal

If you believe that this document breaches copyright please contact us providing details, and we will remove access to the work immediately and investigate your claim 


\title{
Fluxon bunching in supercurrent-coupled Josephson junctions
}

\author{
Niels Grønbech-Jensen and Peter S. Lomdahl \\ Theoretical Division, Los Alamos National Laboratory, Los Alamos, New Mexico 87545 \\ Mogens R. Samuelsen \\ Physics Department, The Technical University of Denmark, 2800 Lyngby, Denmark
}

(Received 29 March 1993)

\begin{abstract}
We investigate analytically and numerically the interaction between fluxons of different Josephson junctions coupled through Cooper-pair tunneling. We find that the supercurrent interaction gives rise to attraction between fluxons regardless of their polarity, although fluxons of different polarity are found to interact with a binding energy of an order of magnitude larger than between unipolar fluxons. When bias current is applied to a system of two coupled junctions we can analytically evaluate the range in the difference of the bias currents for which fluxons are bound. The differences in interaction between fluxons of equal and opposite polarity are discussed. Numerical simulations of coupled sine-Gordon equations agree very well with the analytical predictions.
\end{abstract}

Synchronization of nonlinear oscillations is an important subject in physics and engineering since the collective motion may have essentially different features when compared to motion of the individual oscillator. An example is the emitted electromagnetic power from an array of Josephson junctions. For small junctions this power has been shown to follow the "superradiant" theory and increase with the square of the number of participating oscillators. $^{1,2}$ For junctions with spatial extension, the emitted power has been shown to be able to exceed even this limit ("hyperradiance") (Refs. 3-5) if the oscillators are operated in their fluxon modes. It is therefore important to understand the nature of the different interaction mechanisms possible for these types of oscillators. In this paper we will investigate how a coupling of the supercurrents in two junctions affects the motion of fluxons. This type of coupling has previously been investigated numerically as well as experimentally ${ }^{6}$ for a system of two small Josephson junctions.

We consider the normalized Lagrangian density of two coupled one-dimensional sine-Gordon (SG) systems of the variables $\phi_{1}$ and $\phi_{2}$ :

$$
\begin{array}{r}
\mathcal{L}=\sum_{i=1,2}\left[\frac{1}{2}\left(\phi_{i, t}^{2}-\phi_{i, x}^{2}\right)-1+\cos \phi_{i}\right] \\
-\Delta\left[1-\cos \left(\sum_{i=1,2} \phi_{i}\right)\right] .
\end{array}
$$

Here $x$ is the spatial dimension normalized to the Josephson penetration depth, $t$ is time normalized to the inverse plasma frequency of the junctions, and $\phi_{i}$ is the phase difference between the two superconductors' defining junction $i$. The parameter $\Delta$ is the critical current of the coupling, normalized to the critical current of each junction. From Eq. (1) we get the field equations for $\phi_{i}$ in the form where $i=1,2$. The energy of this system is given by

$$
\begin{gathered}
\mathcal{H}=\int\left\{\sum_{i=1,2}\left[\frac{1}{2}\left(\phi_{i, x}^{2}+\phi_{i, t}^{2}\right)+1-\cos \phi_{i}\right]\right. \\
\left.+\Delta\left[1-\cos \left(\sum_{i=1,2} \phi_{i}\right)\right]\right\} d x .
\end{gathered}
$$

Introducing the usual perturbations to the system, we can write the coupled set of perturbed SG equations as

$$
\phi_{i, x x}-\phi_{i, t t}-\sin \phi_{i}=\Delta \sin \left(\sum_{j=1,2} \phi_{j}\right)+\alpha \phi_{i, t}-\eta_{i}
$$

where $\alpha$ is a dissipative constant representing tunneling of quasiparticles, and $\eta_{i}$ is the normalized bias current through junction $i$. Note that for $\alpha=\eta_{i}=0$ this system has been studied previously. ${ }^{7-9}$ For simplicity here we have chosen to represent the individual junctions with identical physical parameters, i.e., the Josephson lengths, plasma frequencies, critical currents, and the damping parameters are identical for the two junctions. We have represented these parameter differences through the individual bias currents, $\eta_{i}$. This is obviously a reduction of the true parameter space, but it allows us to make some analytical considerations of the dynamics when the symmetry between the equations is broken by one parameter only.

Using the adiabatic perturbation theory ${ }^{10}$ we will study the kink soliton, which for the unperturbed system [left-hand side of Eq. (4)] is given by 


$$
\phi_{i}^{(s)}=4 \tan ^{-1} \exp \left\{\sigma_{i} \gamma\left(u_{i}\right)\left[x-\xi_{i}(t)\right]\right\}
$$

where $\xi_{i}$ is the center of the kink, $u_{i}=\dot{\xi}_{i}$ is its velocity, $\gamma\left(u_{i}\right)=\left(1-u_{i}^{2}\right)^{-1 / 2}$ is the inverse Lorentz contraction, and $\sigma_{i}= \pm 1$ is the polarity.

The existence of bound states between fluxons of different systems can easily be confirmed from the following considerations. Let us assume that $\alpha=\eta_{i}=0$ and that the two fields are given by

$$
\begin{aligned}
& \phi_{1}=\phi_{1}^{(s)}+\mu, \\
& \phi_{2}=\phi_{2}^{(s)}+\nu,
\end{aligned}
$$

where $u_{i}=\xi_{i}=0, \sigma \equiv \sigma_{1} \sigma_{2}=-1$, and $|\mu|,|\nu| \ll 1$. Following Refs. 11 and 12 we will write the dynamical equations for the small deviations, $\mu$ and $\nu$, in the form

$$
\begin{aligned}
& (\mu-\nu)_{x x}-(\mu-\nu)_{t t}=(\mu-\nu) \cos \phi^{(s)}, \\
& (\mu+\nu)_{x x}-(\mu+\nu)_{t t}=(\mu+\nu) \cos \phi^{(s)}+2 \Delta(\mu+\nu) .
\end{aligned}
$$

Looking for oscillating solutions we will substitute the operator $\partial^{2} / \partial t^{2} \rightarrow-\omega^{2}$. The first of the above equations then gives the "translation mode" ${ }^{11}$ frequency $\omega^{2}=0$. The second, Eq. (9), is easily found to give

$$
\omega^{2}=2 \Delta \text { for } \sigma=-1 .
$$

Changing the relative polarity of the kinks from being opposite, $\sigma=-1$, to being equal, $\sigma=1$, we obtain a somewhat different situation,

$$
\begin{aligned}
(\mu-\nu)_{x x}-(\mu-\nu)_{t t}= & (\mu-\nu) \cos \tilde{\phi}^{(s)} \\
(\mu+\nu)_{x x}-(\mu+\nu)_{t t}= & (\mu+\nu) \cos \tilde{\phi}^{(s)} \\
& +2 \Delta \cos 2 \tilde{\phi}^{(s)}(\mu+\nu),
\end{aligned}
$$

where $\tilde{\phi}^{(s)}$ is the kink solution to the double sine-Gordon equation $^{13}$ defined by Eq. (4) for $\phi_{1}=\phi_{2}$. We will, however, use the kink profile of Eq. (5) since we only are interested in an estimate of the bound state frequency to lowest order in $\Delta$. We now have the translation mode frequency, $\omega=0$, given by Eq. (12) and the bound state frequency is then found from Eq. (11) in the following way:

$$
\begin{aligned}
\omega^{2} \int\left(\phi_{x}^{(s)}\right)^{2} d x & =-2 \Delta \int\left(\phi_{x}^{(s)}\right)^{2} \cos 2 \phi^{(s)} d x \\
& \Rightarrow \omega^{2}=\frac{2}{15} \Delta \text { for } \sigma=1 .
\end{aligned}
$$

From Eqs. (10) and (13) we see that bound states $\left(\omega^{2}>\right.$ $0)$ exist for both unipolar and antipolar pairs of fluxons, although the antipolar state has a larger interaction force.

When the system is perturbed by the right-hand side of Eq. (4) we will write the field containing one soliton in the following way:

$$
\phi_{i}=\phi_{i}^{(0)}+\phi_{i}^{(s)}
$$

where $\phi_{i}^{(0)}$ is the solution to Eq. (4) far from the solitons,

$$
\sin \phi_{i}^{(0)}=\eta_{i}-\Delta \sin \left(\sum_{j=1,2} \phi_{j}^{(0)}\right) .
$$

Here we have assumed that there are no spatial nor temporal variations in the background fields. Separating the energy into an unperturbed part, $\mathcal{H}^{(0)}$, and an interaction energy, $\mathcal{H}^{(1)}$, in the form

$$
\begin{aligned}
& \mathcal{H}^{(0)}=\int\left(\sum_{i=1,2}\left[\frac{1}{2}\left(\phi_{i, x}^{2}+\phi_{i, t}^{2}\right)+1-\cos \phi_{i}\right]\right) d x \\
& \mathcal{H}^{(1)}=\int \Delta\left[1-\cos \left(\sum_{i=1,2} \phi_{i}\right)\right] d x
\end{aligned}
$$

we will consider $\mathcal{H}^{(1)}$ to reflect all effects of the coupling, and hence consider $\mathcal{H}^{(0)}$ to be a function of the kink velocities only. This is not exactly true, since the soliton profiles change as a function of $\Delta$. However, for a weak interaction we will see that this is not important.

Inserting Eq. (14) into Eq. (17) and using the unperturbed soliton profiles Eq. (5), we can obtain the interaction energy in the following form:

$$
\begin{aligned}
\mathcal{H}^{(1)} & =\Delta\left(\cos \left(\phi_{1}^{(0)}+\phi_{2}^{(0)}\right) \int\left[1-\cos \left(\phi_{1}^{(s)}+\phi_{2}^{(s)}\right)\right] d x+\sin \left(\phi_{1}^{(0)}+\phi_{2}^{(0)}\right) \int \sin \left(\phi_{1}^{(s)}+\phi_{2}^{(s)}\right) d x\right) \\
& =\Delta\left\{\cos \left(\phi_{1}^{(0)}+\phi_{2}^{(0)}\right) h_{\sigma}+\sin \left(\phi_{1}^{(0)}+\phi_{2}^{(0)}\right) g_{\sigma}\right\}
\end{aligned}
$$

where

$$
\begin{aligned}
& h_{+1}=8 \gamma^{-1}(u) \frac{\cosh [\delta \gamma(u)]+1}{\cosh [\delta \gamma(u)]-1}\left(1-\frac{\delta \gamma(u)}{\sinh [\delta \gamma(u)]}\right), \\
& h_{-1}=8 \gamma^{-1}(u) \frac{\cosh [\delta \gamma(u)]-1}{\cosh [\delta \gamma(u)]+1}\left(1+\frac{\delta \gamma(u)}{\sinh [\delta \gamma(u)]}\right), \\
& g_{+1}=0, \\
& g_{-1}=4 \pi \gamma^{-1}(u) \frac{\sigma_{2}-\sigma_{1}}{2} \frac{\sinh [\delta \gamma(u) / 2]}{\cosh ^{3}[\delta \gamma(u) / 2]},
\end{aligned}
$$

where $\delta=\xi_{1}-\xi_{2}$. In order to evaluate the integrals in Eq. (18) we have assumed that $\left|u_{1}\right|=\left|u_{2}\right|=|u|$. Note that the frequencies given in Eqs. (10) and (13) can also be found from the curvature of $h_{ \pm 1}$ for $\delta=u=0 .^{7}$

Following the procedure outlined in Ref. 12 we define the momentum of system $i$ as

$$
P_{i}=-\int \phi_{i, x} \phi_{i, t} d x
$$

which for the solution Eq. (5) gives

$$
P_{i}^{(s)}=8 u_{i} \gamma\left(u_{i}\right) .
$$

When only looking at localized solutions we may now write the time derivative of the momentum in the following way: 
$\dot{P}_{i}=\Delta \int \phi_{i, x} \sin \left(\phi_{1}+\phi_{2}\right) d x-\alpha P_{i}-\eta_{i} \int \phi_{i, x} d x$.

Inserting Eqs. (2) and (5) into Eq. (25) the following two conditions for a bound state arise:

$\dot{P}_{1}+\dot{P}_{2}=-\alpha\left(P_{1}+P_{2}\right)-2 \pi\left(\sigma_{1} \eta_{1}+\sigma_{2} \eta_{2}\right)$,

$\dot{P}_{1}-\dot{P}_{2}=-\alpha\left(P_{1}-P_{2}\right)-2 \pi\left(\sigma_{1} \eta_{1}-\sigma_{2} \eta_{2}\right)-2 \Delta \frac{\partial}{\partial \delta} \mathcal{H}^{(1)}$.

Since the momentum of a system containing one soliton is determined by its velocity only, Eq. (24), the condition for a bound state can be written

$$
\begin{aligned}
\alpha P & =\pi\left(\sigma_{1} \eta_{1}+\sigma_{2} \eta_{2}\right), \\
\Delta \frac{\partial}{\partial \delta} \mathcal{H}^{(1)} & =\pi\left(\sigma_{1} \eta_{1}-\sigma_{2} \eta_{2}\right),
\end{aligned}
$$

where $P=P_{i}$. The first of these equations gives the power balance velocity of the bound state. The second gives the internal force between the two fluxons. Seeking an estimate of the maximum difference in the bias current, for which the bound state can exist, we find from Eqs. (15), (18), (21), and (22) that to first order in the coupling parameter the asymmetric part, $g_{\sigma}$, of the interaction energy has no importance. The maximum value of the internal force is then numerically found to be given by

$\left.\frac{\partial}{\partial \delta} h_{+1}\right|_{\max } \approx 0.67792945$ for $\delta_{0} \approx 2.1438391 \gamma^{-1}(u)$

$\left.\frac{\partial}{\partial \delta} h_{-1}\right|_{\max } \approx 4.93090430$ for $\delta_{0} \approx 1.0319582 \gamma^{-1}(u)$,

and the locking range in the bias current is then given up to first order in $\Delta$ :

$$
\begin{aligned}
\sigma_{1} \eta_{1}-\sigma_{2} \eta_{2} & =\left.\frac{\Delta}{\pi} \cos \left(\phi_{1}^{(0)}+\phi_{2}^{(0)}\right) \frac{\partial}{\partial \delta} h_{\sigma}\right|_{\max } \\
& \left.\approx \frac{\Delta}{\pi}\left\{1-\frac{1}{2}\left(\eta_{1}+\eta_{2}\right)^{2}\right\} \frac{\partial}{\partial \delta} h_{\sigma}\right|_{\max },
\end{aligned}
$$

where the last equality is exact for $\eta_{1}=\eta_{2}$. Here it is interesting to note that the internal force, and therefore the locking range in the bias current, is constant as a function of the fluxon velocity. The only deviation from the constant comes from the background field, which for $\sigma=1$ makes the interaction force change sign for $\eta_{1}+$ $\eta_{2}=\sqrt{2}$.

Numerical simulations of the system has been performed to understand the dynamical behavior and the limitations to the perturbation results above. We have imposed periodic boundary conditions on the sineGordon systems in order to simulate an infinite system. The numerical procedure for finding the locking range in the bias current was as follows. The system was started with one fluxon trapped in each system with identical parameters. Then after a predefined transient time we determined whether the bound state existed and, if so, we increased the difference in bias so that the average bias was unchanged. This procedure was repeated until the bound state disappeared. In Fig. 1 we show the results of such experiments. The error bars represent the last value of the bias difference (lower point) where the bound state existed and the first value (upper point) where the bound state disappeared. The solid lines are the analytical predictions given by Eqs. (30)-(32). It is evident from Fig. 1 that the agreement between the numerical experiments and the analytical results is excellent for small values of the average bias, $\left(\sigma_{1} \eta_{1}+\sigma_{2} \eta_{2}\right) / 2$. However, for larger values of the bias, we encounter some deviation for all the tried parameter sets: $\diamond, \alpha=0.1$ and $\Delta=0.01 ; \times$, $\alpha=0.05$ and $\Delta=0.01 ;+, \alpha=0.1$ and $\Delta=0.02$. In general, we can only trust the analytical treatment for small values of the perturbations, and hence we should not be surprised to find some deviation for larger bias currents. An interesting observation is to note that for $\sigma=1$ [Fig. 1(a)] the sign change of the coupling seems to agree with the numerical results. In order to under-
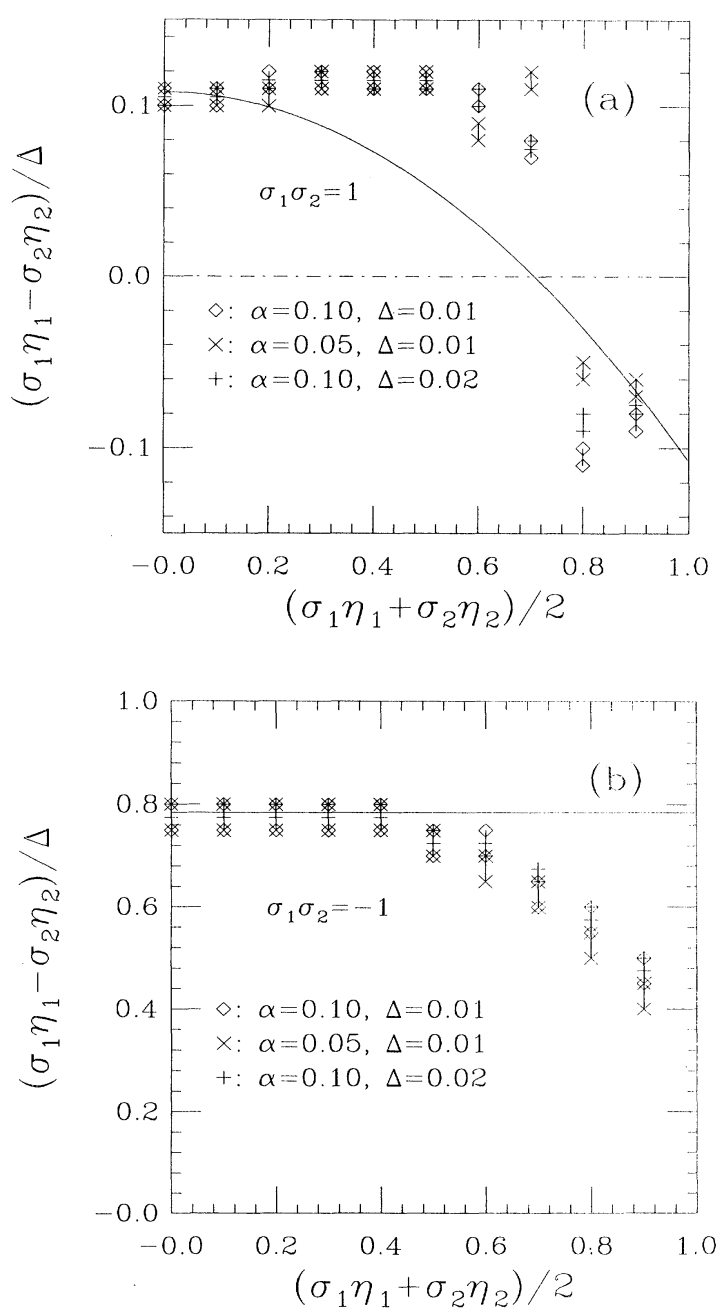

FIG. 1. The locking range in the bias current as a function of the average bias. The solid curve represents the analytical prediction given by Eq. (32). The markers represent the results of the numerical simulations: $\diamond, \alpha=0.1$ and $\Delta=0.01$; $\times, \alpha=0.05$ and $\Delta=0.01 ;+, \alpha=0.1$ and $\Delta=0.02$. 
stand the origin of the deviation, we have calculated the different components of Eq. (27) numerically. The term $-\alpha\left(P_{1}-P_{2}\right)$ has been calculated from Eq. (23) and the internal force term has been calculated in the following form:

$-2 \Delta \frac{\partial}{\partial \delta} \mathcal{H}^{(1)}=\int\left(\phi_{1, x}-\phi_{2, x}\right) \sin \left(\phi_{1}+\phi_{2}\right) d x$.

As argued above, the perturbation theory has assumed that the difference in momenta is zero for the bunched state, since the perturbation expression for the momentum of a fluxon is given by the velocity alone. However, it is known ${ }^{14}$ that the true momentum also depends on the bias current in a non trivial way, and we can therefore expect the difference in momentum of the two lines to differ from zero. That is exactly what happens for larger values of the bias current.

In Fig. 2 we have shown the internal force $(+)$ and the difference in momenta $(x)$ calculated from the numerically evaluated wave profiles in case of $\sigma=1$. For low values of the bias currents, $\left(\sigma_{1} \eta_{1}+\sigma_{2} \eta_{2}\right) / 2=0.1$ [Fig. 2(a)], we find that the difference in momenta (multiplied by the dissipation) is vanishing compared to the interaction force, and the predicted locking range is then in very good agreement with the numerical results [Fig. 1(a)]. However, for increasing values of the bias current [Fig. 2(b)] we find that the difference in momenta is not insignificant compared to the internal force. Since the locking range is determined by the sum of the two $(\diamond)$, the analytical result based only on the internal force may now deviate from the numerical results. This becomes even more evident when looking at Fig. 2(c), where we have shown the
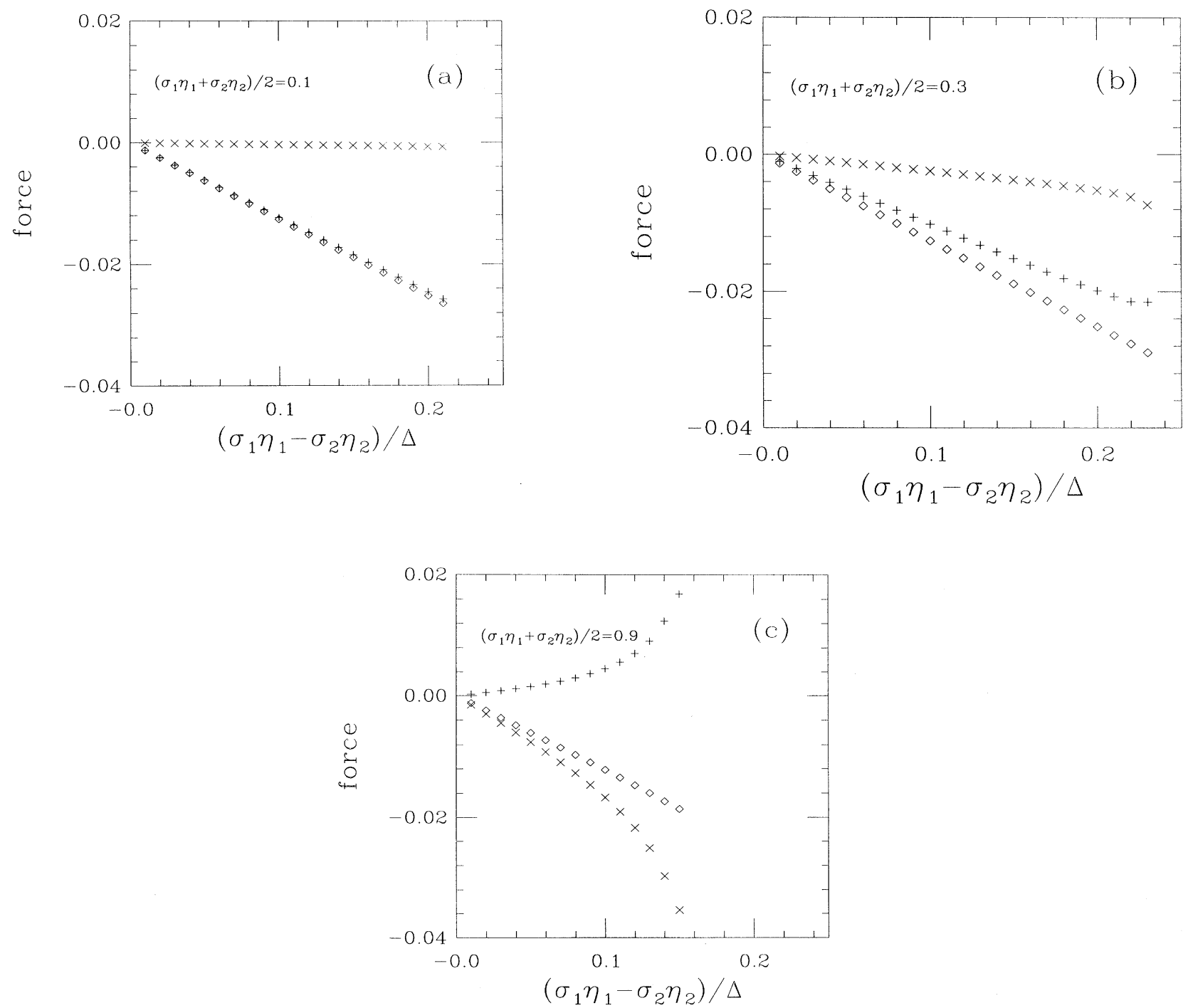

FIG. 2. The internal force $(+)$ given by Eq. (33) and the difference in momenta times the dissipation $(\times)\left[-\alpha\left(P_{1}-P_{2}\right)\right]$ calculated from Eq. (23) as a function of the difference in the bias current, $\sigma_{1} \eta_{1}-\sigma_{2} \eta_{2}$. Both quantities are calculated from the numerically obtained wave profiles. The $\diamond$ is the sum of + and $\times . \sigma=1$. 
force components for $\left(\sigma_{1} \eta_{1}+\sigma_{2} \eta_{2}\right) / 2=0.9$. Here we see the peculiar situation that the internal force has changed sign (repulsion) as predicted by the perturbation theory Eq. (32). However, since the difference in the momenta is now the dominating of the two contributions to the locking range we still find an effective attraction between the fluxons ( $\diamond$ negative).

Similar to this we can demonstrate that the deviation for large bias values in Fig. 1(b) $(\sigma=-1)$ is due to an increasing difference in the momenta as a function of the average bias. Again, for low values, $\left(\sigma_{1} \eta_{1}+\sigma_{2} \eta_{2}\right) / 2=0.1$ [Fig. 3(a)], we find almost exact zero for the momentum difference, whereas for a large bias, $\left(\sigma_{1} \eta_{1}+\sigma_{2} \eta_{2}\right) / 2=$ 0.9 [Fig. 3(b)], we find a significant contribution from
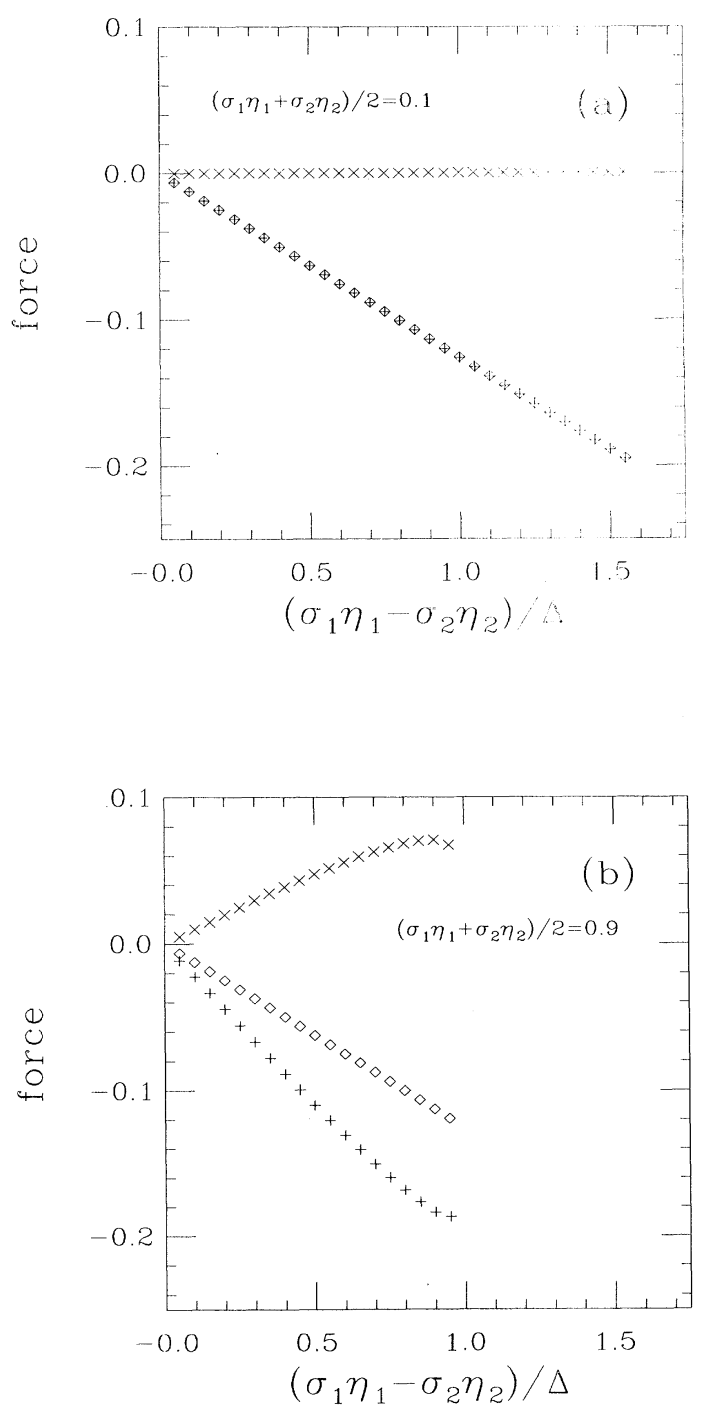

FIG. 3. The internal force (+) given by Eq. (33) and the difference in momenta times the dissipation $(\times)\left[-\alpha\left(P_{1}-P_{2}\right)\right]$ calculated from Eq. (23) as a function of the difference in the bias current, $\sigma_{1} \eta_{1}-\sigma_{2} \eta_{2}$. Both quantities are calculated from the numerically obtained wave profiles. The $\diamond$ is the sum of + and $\times . \sigma=-1$.

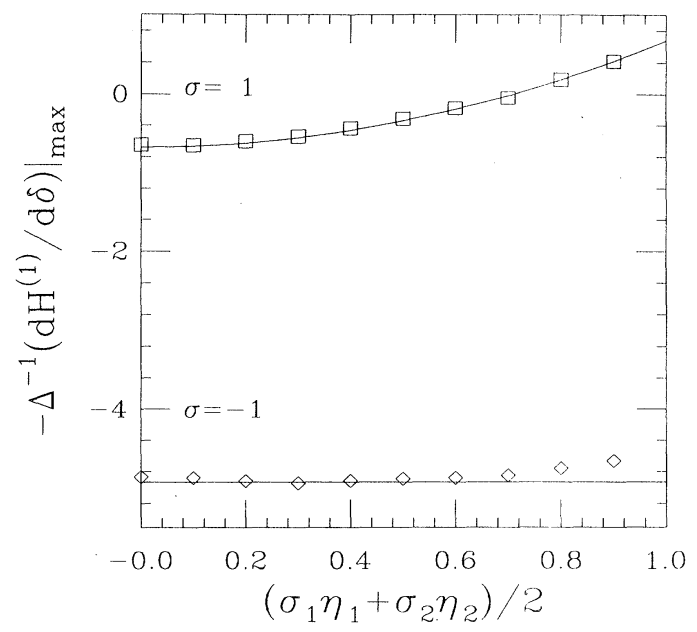

FIG. 4. The extreme of the internal force $(+)$ given by Eq. (33) as a function of the average in the bias current, $\left(\sigma_{1} \eta_{1}+\right.$ $\left.\sigma_{2} \eta_{2}\right) / 2$. Solid lines are the analytical result given by Eq. (34) and the $\square$ represents the obtained values from the numerical experiments. $\alpha=0.1$ and $\Delta=0.02$.

the difference in momenta, resulting in a smaller locking range than predicted.

It is interesting to observe that the deviation observed in Fig. 1 between the numerical results and the analytical prediction is solely due to the assumption of $P_{1}=P_{2}$ for the bound state. This becomes clear when looking at Fig. 4. Here we have shown the maximum interaction force calculated from Eq. (33) as a function of the bias current. The solid lines represent Eq. (33) when the analytical value

$$
-\left.\Delta \frac{\partial}{\partial \delta} h_{\sigma}\right|_{\max }\left\{1-\frac{1}{2}\left(\eta_{1}+\eta_{2}\right)^{2}\right\}
$$

is used, and the squares represent the most extreme values found from the numerical experiments for $\Delta=0.02$ and $\alpha=0.1$ (see Figs. 1-3). The agreement is evidently almost perfect for all bias values and hence we will conclude that the interaction force is very well represented by the analytical expression.

We have investigated the interaction between solitons of different sine-Gordon systems in the presence of a nonlinear coupling mechanism. For Josephson junctions this interaction corresponds to a coupling in the supercurrent. We have studied the condition for bound states between magnetic fluxons for different values of the bias current and the difference between the unipolar and antipolar fluxon states has been discussed. Very good agreement between the analytical and numerical results has been obtained and the origin of the discrepancies has been studied in detail. For Josephson junctions it may not be experimentally possible to study the supercurrent coupling isolated. Two different layouts of junctions that could produce the supercurrent coupling have been published; the adjacent geometry, ${ }^{3}$ and the stacked geometry ${ }^{15}$ (diagrams showing the geometries can be found in Refs. 3 
and 15). Both these geometries result in an inductive coupling mechanism as well. It is therefore important to consider all the different coupling mechanisms at once if one will make direct comparisons to experiments. Since the strengths of the different couplings depend on the specific geometry of an experimental device, we have cho- sen to investigate the coupling in the supercurrent separately from other types of interactions (inductive and capacitive). ${ }^{12}$

This work was performed under the auspices of the US Department of Energy.
${ }^{1}$ D. R. Tilley, Phys. Lett. 33A, 205 (1970).

${ }^{2}$ T. F. Finnegan and S. Wahlstein, Appl. Phys. Lett. 21, 541 (1972).

${ }^{3}$ T. Holst, J. B. Hansen, N. Grønbech-Jensen, and J. A. Blackburn, Phys. Rev. B 42, 127 (1990); IEEE Trans. Mag. 27, 2704 (1991).

${ }^{4}$ M. Cirillo, I. Modena, F. Santucci, P. Carelli, and R. Leoni, Phys. Lett. A 167, 175 (1992).

${ }^{5}$ Niels Grønbech-Jensen and James A. Blackburn, Phys. Rev. Lett. 70, 1251 (1993).

${ }^{6}$ H. J. T. Smith and M. Dion, Phys. Rev. B 42, 206 (1990).

${ }^{7}$ Yuri S. Kivshar, J. Phys. Soc. Jpn. 57, 4232 (1988).

${ }^{8}$ O. M. Braun, Yu. S. Kivshar, and A. M. Kosevich, J. Phys.
C 21, 3881 (1988).

${ }^{9}$ Yuri S. Kivshar and Boris A. Malomed, Rev. Mod. Phys. 61, 763 (1989).

${ }^{10}$ D. W. Mclaughlin and A. C. Scott, Phys. Rev. A 18, 1652 (1978).

${ }^{11}$ J. Rubinstein, J. Math. Phys. 11, 258 (1970).

${ }^{12}$ N. Grønbech-Jensen, M. R. Samuelsen, P. S. Lomdahl, and J. A. Blackburn, Phys. Rev. B 42, 3976 (1990).

${ }^{13}$ T. Uchiyama, Phys. Rev. D 14, 3520 (1976).

${ }^{14}$ O. A. Levring, M. R. Samuelsen, and O. H. Olsen, Physica D 11, 987 (1984).

${ }^{15}$ M. B. Mineev, G. S. Mkrtchyan, and V. V. Schmidt, J. Low Temp. Phys. 45, 497 (1981). 